\section{Maladies}

\title{
Du nouveau sur la chorée de Huntington
}

L'identification du gène $I T_{15}$ il y a maintenant trois ans [1] était l'annonce d'avancées nouvelles et rapides dans la compréhension des mécanismes de la maladie et d'un effort renouvelé de recherches à visée thérapeutique. Le Congrès de la Society for Neuroscience (San Diego, novembre 1995) a été l'occasion de faire le point, en même temps que sortaient, ou étaient annoncés, plusieurs articles importants.

La huntingtine est largement distribuée dans le système nerveux

Le groupe mené par Christopher Ross à Johns Hopkins (Baltimore, MD, USA) avait, au mois de mai dernier, publié la localisation de la protéine dans le système nerveux central [2] grâce à deux anticorps reconnaissant la région aminoterminale, d'une part, un épitope interne, d'autre part. Dans les deux cas, la protéine reconnue avait $350 \mathrm{kDa}$ et était largement - et assez uniformément - distribuée dans le système nerveux. Dans les neurones, la protéine était présente dans tous les compartiments en dehors du noyau et des mitochondries. Elle semblait un peu plus associée aux terminaisons qu'aux autres compartiments neuronaux.

L'équipe conduite par Marian DiFiglia au Massachusetts General Hospital (Boston, MA, USA) arrive à des conclusions très voisines [3-6] en utilisant trois autres anticorps qui reconnaissent d'autres régions de la molécule. L'analyse de synaptosomes provenant de tissus corticaux a permis à ce groupe d'associer la huntingtine non mutée à des protéines de la membrane vésiculaire comme $\mathrm{SV}_{2}$, le récepteur de la transférine ou la synaptophysine. La huntingtine étant distribuée largement dans les neurones - et non concentrée dans les terminaisons, pour ces auteurs - c'est un rôle dans le trafic vésiculaire que cette localisation pourrait traduire. Un rôle certainement essentiel au développement et à la survie car des souris dont le gène $I T_{15}$ a été invalidé sont incapables de survivre à l'état homozygote au-delà du $9^{\mathrm{e}}$ jour intrautérin, stade précédant la neurulation mais auquel les trois feuillets sont déjà individualisés $[7,8]$. Les hétérozygotes montrent des déficits comportementaux (hyperactivité) relativement modérés associés à des pertes neuronales localisées qui indiquent que la molécule, malgré sa large distribution homogène, peut jouer un rôle plus important dans certaines populations neuronales que dans d'autres. Il ne s'agit pas, toutefois, des groupes neuronaux atteints chez les patients, ce qui limite un peu les conclusions que l'on peut tirer de ces expériences.

\section{La huntingtine mutée est synthétisée}

Trois larges groupes collaboratifs ont démontré, cette année, que la molécule contenant une séquence accrue de glutamines $(\geq 40)$ - pendant de la présence des triplets CAG dans la région 5' codante d' $I T_{15}$ - est effectivement synthétisée chez les patients hétérozygotes. Cela appuie fortement l'hypothèse de travail commune selon laquelle la maladie est liée à un gain de fonction $\left(\mathrm{m} / \mathrm{s} n^{\circ} 6, v o l .11\right.$, p. 919).

Les groupes de Christopher Ross et de Marian DiFiglia ont montré par immunoélectrophorèse l'apparition d'une huntingtine de poids molécu- laire plus élevé chez les patients. Les anticorps utilisés reconnaissaient tous, cependant, des régions non mutées de la molécule.

L'équipe de Jean-Louis Mandel (Inserm, Strasbourg), en collaboration avec celle d'Yves Agid (Inserm, Paris), vient de créer un outil qui permet d'effectuer directement et spécifiquement, l'identification de la protéine mutée, sous la forme d'un anticorps monoclonal (1C2) qui reconnaît des séries polyglutaminiques et s'y associe d'autant plus fortement que cette série est longue [9]. Cet anticorps, qui permet d'identifier aussi les polyglutamines répliquées dans les ataxines associées à certaines ataxies spino-cérébelleuses, sera certainement très important, non seulement pour étudier les mécanismes de la chorée de Huntington mais aussi pour aller chercher d'autres modifications de chaînes polyglutaminiques dans d'autres maladies dont les défauts moléculaires sont encore inconnus.

\section{HAP-1, molécule de l'année !}

Le congrès $[10,11]$ et, juste après, une publication dans Nature [12] ont permis à l'équipe de Christopher Ross de présenter une nouvelle molécule dont le moins que l'on puisse dire est qu'elle ouvre des perspectives. La question à laquelle HAP-1 (pour Huntington-associated protein 1) pourrait apporter un début de réponse - en attendant HAP-2, 3, etc. - est celle de l'effet délétère d'un accroissement de la chaîne de polyglutamines. Le clone trouvé, parmi 100000 , contenait une insertion de 507 paires de base (sans homologie avec un gène connu) qui codait pour

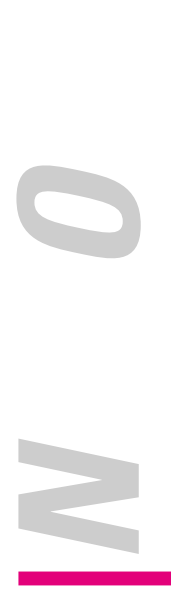


une séquence d'acides aminés s'associant avec la huntingtine dans des expériences de double-hybrides. Or la force de cette interaction, présente que la huntingtine soit mutée ou non, semble dépendre directement de la longueur de la chaîne de polyglutamines. A partir de l'ADNc partiel, le criblage de banques de rat a permis d'identifier deux protéines (rHAP1-A et B) et d'obtenir par PCR, à partir de ces séquences, un fragment de la protéine humaine hHAP1 très proche de celle du rat (96\% d'identité des acides aminés pour le fragment considéré).

Une donnée importante supplémentaire qu'apporte l'étude d'HAP1 chez le rat est celle de la localisation car, au contraire de la huntingtine, la protéine associée n'est présente que dans le cerveau, notamment dans les régions qui, comme le noyau caudé ou le cortex cérébral, sont fortement touchées par la maladie.

L'hypothèse qui commence à prendre corps autour de l'identification de HAP-1 est que l'effet délétère de la huntingtine mutée ne toucherait que des cellules dans lesquelles elle interagirait spécifiquement avec certaines molécules. La synthèse quasi ubiquitaire de la huntingtine ne permettant pas d'interpréter les mécanismes qui conduisent à la dégénérescence neuronale dans le striatum, peut-être celle (bien plus restreinte) d'HAP-1 - ou d'une autre protéine associée - le permettraitelle ? On ne peut qu'être frappé, à ce stade, de la vitesse à laquelle avance cette recherche "après-gène ", qui rappelle celle des découvertes qui ont succédé à l'identification de la dystrophine. Le parallèle souligne, toutefois, combien le chemin peut être long entre ces travaux fondamentaux, même accélérés, et la mise au point de thérapeutiques.

\section{Modèles et traitements expérimen- taux}

Le Congrès des Neurosciences a permis, sur ce point, la présentation de plusieurs travaux importants (dont la publication est en cours dans $\mathrm{J}$. Neuroscience) par les équipes de Flint Beal du Massachusetts General Hospital
(Boston, MA, USA) et de Philippe Hantraye du Service Hospitalier Frédéric-Joliot (Orsay, France) qui, d'ailleurs, collaborent étroitement.

Flint Beal a présenté lors du congrès une revue approfondie sur les mécanismes potentiels d'atteinte neuronale provoquée par un défaut du métabolisme mitochondrial, et sur les traitements existants ou hypothétiques permettant de s'attaquer à chacun de ces mécanismes [13]. Il a également apporté une nouvelle preuve à l'appui de l'hypothèse d'une atteinte du métabolisme énergétique chez les malades grâce à une étude spectroscopique montrant l'effondrement des complexes II et III mitochondriaux dans le striatum des patients et la préservation, au contraire, des complexes I et IV dans les mêmes zones [14]. De façon intéressante, ces auteurs ont observé que l'atteinte du striatum était sélective car cet effondrement n'existait pas dans le cortex cérébral ou le cervelet. Les traitements envisagés comprennent, en particulier, des bloquants de la libération de glutamate, comme le Riluzole ${ }^{\circledR}$ de Rhône-Poulenc Rorer, la lamotrigine et le BW 1003C87, qui sont capables de diminuer l'étendue des zones de dégénérescence induites par des toxines agissant sur les complexes mitochondriaux comme le $\mathrm{MPP}^{+}$ou l'acide 3nitropropionique (3-NP). Une seconde classe est formée d'antagonistes de récepteurs NMDA comme la mémantine, qui réduit l'effet toxique du malonate. On trouve ensuite des agents protecteurs directs ou indirects - contre l'effet des radicaux libres oxygénés comme le coenzyme Q10 ou le n-tert-butyl-a(2 sulfophenyl)-nitrone (S-PBN). Parmi les essais réalisés par l'équipe de Flint Beal, deux retiennent l'attention. D'une part, l'association de plusieurs de ces substances semble potentialiser leur action. Celle du MK-801 (antiglutamate) et du coenzyme Q10 pourrait autoriser leur utilisation à doses faibles, non toxiques. Il pourrait en être de même pour l'association nicotinamide/S-PBN. La combinaison d'agents capables d'interférer avec des mécanismes induisant une dégénérescence à des niveaux différents de la cas- cade d'événements est, sans doute, une voie riche de promesses. Le second essai, plus achevé, concerne un bloquant spécifique de la NO-synthase (NOS) neuronale, le 7-nitroindazole (7-Ni). L'administration systémique de 7-Ni chez des rats soumis à l'injection de 3-NP a permis une protection quasi complète du striatum. L'analyse biochimique du tissu a montré que l'inhibition de la NOS bloquait significativement la formation de composés comme la 3-nitrotyrosine qui découle de la synthèse de peroxynitrite. L'inhibition de la synthèse de NO jouerait donc clairement, dans ce cas, un rôle protecteur. La toxicité d'un inhibiteur spécifique de la NO synthase neuronale étant faible, aux doses utilisées, la confirmation de ces résultats dans un modèle expérimental plus proche de l'homme est très attendue.

C'est à cette tâche - entre autres que s'est attaquée l'équipe de Philippe Hantraye qui, cette année, a largement exposé le modèle d'intoxication chronique par le 3-NP de babouins adultes, et montré ses relations avec la maladie de Huntington tant sur le plan comportemental (effets moteurs et cognitifs) que sur le plan histologique (lésions striatales spécifiques, en gradient évolutif) [1517]. Ce modèle, développé chez le babouin et à présent étendu au macaque, représente certainement l'outil le plus efficace à ce jour pour envisager des traitements potentiels de la maladie [18]. Il s'agit, en effet, d'une atteinte histologique bilatérale qui reproduit la plupart des signes de la maladie, y compris l'existence de tentatives transitoires de régénération neuronale [19]. Cette atteinte s'accompagne de troubles moteurs spontanés (dystonies) ou inductibles (hyperkinésie), et de troubles cognitifs spontanés qui reproduisent la symptomatologie de type frontal de la maladie de Huntington. La disponibilité de ce modèle pour tester les diverses thérapeutiques actuellement envisagées pour lutter contre la maladie de Huntington permettra, on peut l'espérer, de les mettre au point plus rapidement, et dans des conditions de sécurité bien meilleures pour les patients. 
$\mathrm{Au}$ total, on pouvait à l'issue du Congrès de San Diego être optimiste... et impatient quant à l'avenir thérapeutique d'une maladie que l'on apprend à connaître enfin, depuis trois ans, de mieux en mieux et pour laquelle l'arsenal thérapeutique hypothétique semble quasiment pléthorique face à l'absence totale de tout traitement qui était son lot jusqu'à aujourd'hui.

M.P.

1. Huntington's disease collaborative research group. A novel gene containing a trinucleotide repeat that is expanded and unstable on Huntington's disease chromosomes. Cell 1993; 72: 971-83.

2. Sharp AH, Loev SJ, Schilling G, et al. Widespread expression of Huntington's disease gene (IT15) protein product. Neuron 1995; 14: 106574 .

3. DiFiglia M, Sapp E, Chase K, et al. Huntingtin is a cytoplasmic protein associated with vesicles in human and rat brain neurons. Neuron 1995; 14 : 1075-81.
4. Aronin N, Chase K, Young C, et al. CAG expansion affects the expression of mutant Huntingtin in the Huntington's disease brain. Neuron 1995; 15: 1193-1201.

5. Aronin N, Chase K, Young C, et al. Variable expression of the mutant protein of the HD gene in Huntington's disease brain. Neurosci Abst $\mathrm{n}^{\circ} 21$, $1995,198.4$

6. Day M, Schwarz C, Sheth A, et al. Huntingtin is expressed in embryonic mouse and human brain. Neurosci Abst $\mathrm{n}^{\circ}$ 21, 1995, 198.3.

7. Nasir J, Diewert VM, Richman JM, et al. Targeted disruption of the murine Huntington's disease gene I: early post-implantation embryonic lethality in homozygotes. Neurosci Abst n ${ }^{\circ}$ 21, 1995, 685.3.

8 . Floresco SB, Nasir J, O’Kusky JR, et al. Targeted disruption of the murine Huntington's disease gene II : behavioral and neuropathalogical assessment. Neurosci Abst $\mathrm{n}^{\circ}$ 21, 1995, 685.4.

9. Trottier Y, Lutz Y, Stevanin G, et al. Polyglutamine expansion as a pathological epitope in Huntington's disease and four dominant cerebellar ataxias. Nature 1995; 378: 403-5.

10. Li SH, Li XJ, Lanahan A, et al. Cloning of a protein (HAP-1) that associates with the Huntington's disease protein. Neurosci Abst $\mathrm{n}^{\circ}$ 21, 1995, 198.7.

11. Li XJ, Li SH, Sharp AH, et al. A novel protein (HAP-1) enriched in brain interacts with the Huntington's disease protein. Neurosci Abst $\mathrm{n}^{\circ} 21$, 1995, 685.1.
12. Li XJ, Li SH, Sharp AH, et al. A. Huntingtinassociated protein enriched in brain with implications for pathology. Nature 1995; 378: 398-402. 13. Beal MF, Matthews MT, Henshaw DR, et al. Neuroprotective strategies for treatment of lesions produced by mitochondrial toxins : implications for neurodegenerative diseases. Neurosci Abst $\mathrm{n}^{\circ}$ 21, 1995, 685.7.

14. Browne SE, Bowling AC, Mac Garvey U, et al. Oxidative DNA damage and impaired mitochondrial metabolism in Huntington's disease. Neurosci Abst $\mathrm{n}^{\circ}$ 21, 1995, 198.9 .

15. Brouillet E, Palfi S, Ferrante R, et al. Systemic administration of 3-nitropropionic acid in primates: correlation between MRI findings and symptomatology. Neurosci Abst $\mathrm{n}^{\circ}$ 21, 1995, 198.12. 16. Hantraye P, Brouillet E, Palfi S, et al. Chronic 3-nitropropionic acid treatment in baboons: a primate model for Huntington's disease? Neurosci Abst $\mathrm{n}^{\circ} 21,1995,198.10$.

17. Palfi S, Brouillet E, Ferrante RJ, et al. Cognitive and motor deficits in a primate model of Huntington's disease. Neurosci Abst ${ }^{\circ}$ 21, 1995, 198.11. 18. Hantraye P. Maladie de Huntington et greffes intrastriatales de neurones embryonnaires chez le primate infra-humain. médecine/sciences 1992; 8: 852-3.

19. Guyot MC, Hantraye P, Moya K, et al. Abnormal NADPH-diaphorase staining in striatal interneurons of rats chronically treated with 3-nitropropionic acid. Neurosci Abst $\mathrm{n}^{\circ}$ 21, 1995, 198.13.
Apoptose et maladie d'Alzheimer: le lien STM2. Récemment, nous rapportions l'identification de nouveaux gènes impliqués dans des formes sévères de maladies d'Alzheimer familiales à manifestations précoces $\left(m / s n^{\circ} 9\right.$, vol. $\left.11, p .1354\right)$. Cependant, la fonction des deux gènes de la même famille identifiés (S182 et STM2) restait totalement inconnue. Vito et al. (Bestheda, MD, USA) suggèrent maintenant que le gène STM2 pourrait intervenir comme un modulateur du signal apoptotique [1]. Ces auteurs ont cloné, par la technique de sélection fonctionnelle, des ADNc interférant avec l'apoptose de lymphocytes $\mathrm{T}$ induite par la stimulation du récepteur pour l'antigène des cellules $T$ (TCR). Une banque d'expression d'ADNc a été transfectée dans ces lymphocytes T en culture et les clones résistant dès lors à l'apoptose induite par la stimulation des TCR ont été isolés, amplifiés, et l'ADNc responsable de cette résistance a été cloné et analysé ; ces clones étaient appelés $A L G 2$ et ALG3 (apoptosis-linked genes). ALG2 est un clone antisens complémentaire d'un messager codant pour une protéine liant le calcium alors que ALG3 a le potentiel de coder pour un fragment de l'homologue murin du produit du gène STM2. Un vecteur d'expression pour la protéine ALG2 sensibilise les cellules dans lesquelles il est transfecté à l'apoptose, ce qui indique que cette protéine est impliquée dans la transmission du signal apoptotique, et confirme l'importance du calcium dans ce phéno- mène. Le mode d'action du clone ALG3 reste inconnu, puisqu'il ne code que pour une protéine du type STM2 tronquée. Son action au niveau des lymphocytes $\mathrm{T}$ semble double : il bloque l'induction du ligand de Fas et le signal relayé par Fas $\left(m / s n^{\circ} 1\right.$, vol. $\left.12, p .84\right)$. Malgré ces incertitudes sur le mécanisme d'action antiapoptotique de ce clone $A L G 3$, ces résultats renforcent l'idée que STM2 pourrait être normalement impliqué dans le contrôle de l'apoptose, sa mutation étant associée à une sensibilisation des cellules neuronale cibles à l'apoptose.

[1. Vito P, et al. Science 1996; 271: 521-5.]

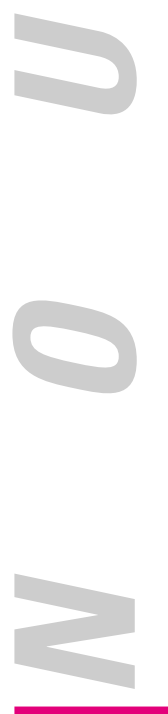

